\title{
ALMACAL IV: a catalogue of ALMA calibrator continuum observations
}

\author{
M. Bonato, ${ }^{1,2 \star}$ E. Liuzzo, ${ }^{1}$ A. Giannetti, ${ }^{1}$ M. Massardi, ${ }^{1}$ G. De Zotti, ${ }^{2}$ S. Burkutean, ${ }^{1}$ \\ V. Galluzzi, ${ }^{3,4}$ M. Negrello, ${ }^{5}$ I. Baronchelli, ${ }^{6}$ J. Brand, ${ }^{1}$ M. A. Zwaan, ${ }^{7}$ K. L. J. Rygl, ${ }^{1}$ \\ N. Marchili, ${ }^{8}$ A. Klitsch ${ }^{7,9}$ and I. Oteo ${ }^{7,10}$ \\ ${ }^{1}$ INAF-Istituto di Radioastronomia, and Italian ALMA Regional Centre, Via Gobetti 101, I-40129 Bologna, Italy \\ ${ }^{2}$ INAF-Osservatorio Astronomico di Padova, Vicolo Osservatorio 5, I-35122 Padova, Italy \\ ${ }^{3}$ INAF-Osservatorio Astronomico di Trieste, Via Tiepolo 11, I-34143 Trieste, Italy \\ ${ }^{4}$ Dipartimento di Fisica e Astronomia, Università di Bologna, via Gobetti 93/2, I-40129 Bologna, Italy \\ ${ }^{5}$ School of Physics and Astronomy, Cardiff University, The Parade, Cardiff CF24 3AA, UK \\ ${ }^{6}$ California Institute of Technology, Pasadena, CA 91125, USA \\ ${ }^{7}$ European Southern Observatory, Karl-Schwarzschild-Str. 2, D-85748 Garching, Germany \\ ${ }^{8}$ INAF-IAPS, Via Fosso del Cavaliere 100, I-00133 Roma, Italy \\ ${ }^{9}$ Department of Physics, Centre for Extragalactic Astronomy, Durham University, South Road, Durham DH1 3LE, UK \\ ${ }^{10}$ Institute for Astronomy, University of Edinburgh, Royal Observatory, Blackford Hill, Edinburgh EH9 3HJ, UK
}

Accepted 2018 April 20. Received 2018 March 26; in original form 2018 February 2

\begin{abstract}
We present a catalogue of ALMA flux density measurements of 754 calibrators observed between 2012 August and 2017 September, for a total of 16263 observations in different bands and epochs. The flux densities were measured by reprocessing the ALMA images generated in the framework of the ALMACAL project, with a new code developed by the Italian node of the European ALMA Regional Centre. A search in the online data bases yielded redshift measurements for 589 sources ( $\sim 78$ per cent of the total). Almost all sources are flat spectrum, based on their low-frequency spectral index, and have properties consistent with being blazars of different types. To illustrate the properties of the sample, we show the redshift and flux density distributions as well as the distributions of the number of observations of individual sources and of timespans in the source frame for sources observed in bands 3 $(84-116 \mathrm{GHz})$ and $6(211-275 \mathrm{GHz})$. As examples of the scientific investigations allowed by the catalogue, we briefly discuss the variability properties of our sources in ALMA bands 3 and 6 and the frequency spectra between the effective frequencies of these bands. We find that the median variability index steadily increases with the source-frame time lag increasing from 100 to $800 \mathrm{~d}$, and that the frequency spectra of BL Lacs are significantly flatter than those of flat-spectrum radio quasars. We also show the global spectral energy distributions of our sources over 17 orders of magnitude in frequency.
\end{abstract}

Key words: galaxies: abundances-galaxies: active-galaxies: photometry-submillimetre: galaxies.

\section{INTRODUCTION}

The Atacama Large Millimeter/submillimeter Array (ALMA) calibrators comprise many hundreds of bright, compact radio sources, distributed over about 85 per cent of the sky.

Every ALMA science project includes observations of calibrator sources (mostly bright quasars in the $\mathrm{mm}$ and sub-mm regime) to set the flux density scale, to measure the band pass response, and to calibrate amplitude and phase of the visibilities of the science targets (Fomalont et al. 2014).

Such observations represent a significant fraction (generally $\lesssim 30$ per cent) of each execution block (EB). If the calibrator is a phase calibrator, it is observed many times during the same EB. If it is a band pass or an amplitude calibrator, it is typically observed once per EB. Therefore, each calibrator can be observed several times, on different dates, in different ALMA bands and array configurations, for one or multiple science projects.

The fields around ALMA calibrators of projects stored in the ALMA Science Archive have been exploited to carry out a novel, wide and deep (sub-)millimetre survey, ALMACAL (Oteo et al. 
2016), and to investigate detected sources of special interest (Klitsch et al. 2017; Oteo et al. 2017). The ALMACAL survey, in fact, takes advantage of the high sensitivity reached in the fields of ALMA calibrator observations to blindly extract a multi band and multiepoch survey of dusty star-forming galaxies. Together with this primary goal, the same observations offer a unique opportunity to investigate spectral behaviour and variability of a large sample of bright extragalactic sources, the calibrators themselves, mostly active galactic nuclei across the whole (sub-)millimetric band.

In this paper, we present a catalogue of observations of the ALMA calibrators collected, so far, for the ALMACAL project purposes. Their multi-epoch, multi frequency measurements over a poorly explored spectral region constitute a rich data base, well-suited for a variety of scientific investigations, some of which will be more extensively detailed in future papers of our collaboration.

This paper is structured as follows. In Section 2, the catalogue is introduced. An account of the source classification is given in Section 3, where we also compare the frequency spectra of BL Lacs and flat-spectrum radio quasars (FSRQs). In Section 4, we describe the main properties of the catalogue and, as an example of its scientific exploitation, we briefly discuss the variability properties of our sources in ALMA bands 3 and 6. In Section 5, we present the global spectral energy distributions (SEDs) of sources, built by collecting data from online data bases. Finally, Section 6 contains a short summary of the paper.

\section{THE SAMPLE}

The catalogue consists of continuum measurements of calibrators, obtained during the majority of the ALMA science observations between 2012 August and 2017 September. In total, we collected 16263 observations $^{1}$ of 754 calibrators. Being a collection of data from a heterogeneous sample of science projects, our observations vary within a wide spectrum of different frequency set-ups, array configurations, and integration times.

The details of calibration and imaging for the ALMACAL data are described by Oteo et al. (2016). Here, we summarize a few pieces of information, useful for a comprehensive description of the presented catalogue. For our purpose, we considered all the ALMA projects in the epochs 2012-2017 and, in them, the extragalactic calibrators at any observing band. The full data deliveries available in the ALMA archive were retrieved for data sets for which the proprietary period had expired, while only the calibrator data were considered for the remaining projects, after an official request through an ALMA Helpdesk ticket. Calibration scripts produced (during the ALMA Quality Assessment procedure) and distributed through the archive were run to generate the calibration tables that were applied to all the calibrators (in some cases differently with respect to what is usually done for the archived data, for which, especially in the first observing cycles, tables were applied only to science targets and phase calibrators). Data were self-calibrated taking advantage of the presence of the calibrator in the phase centre and images were produced with the calibrator present and subtracted (in the visibility domain). The latter are used in the ALMACAL collaboration to investigate the background looking for dusty galaxies. The former are used in this paper to investigate the calibrator population properties.

${ }^{1}$ From the initial sample, we removed $\sim 1.7$ per cent of the images that showed anomalies.
For the calibrators, the flux densities were uniformly measured from the ALMA images ${ }^{2}$ using a new code developed by the Italian node of the European ALMA Regional Centre (ARC). This software is part of a suite of tools aimed at easing the ALMA Science Archive mining: the ALMA Keyword Filler tool package (AKF; Liuzzo et al. 2018) and the Keywords of Astronomical FITSimages Explorer (KAFE; Burkutean et al. 2018). The AKF codes are particularly useful to compare image products or to identify the images to be selected for several scientific purposes. KAFE is a web-based FITS image post-processing analysis tool. It exploits AKF and complements selected FITS files with metadata based on a uniform image analysis approach while also offering advanced image diagnostic plots. KAFE's applicability to multi-instrument images in the radio to sub-mm wavelength domain makes it ideal for data sample studies requiring uniform data diagnostic criteria.

After the estimation of the rms $(\sigma)$ in an image, the code masks the pixels with a flux density below $5 \sigma$ and obtains the source flux density by integrating over the remaining pixels. This is enough to cope with both isolated point-like and extended sources definition, which strongly depend on the observing strategy and phase decoherence, and might vary for our targets from one observation to the other.

The number of observations in the different ALMA bands are 5100 in band $3(84-116 \mathrm{GHz}), 639$ in band $4(125-163 \mathrm{GHz})$, 6319 in band $6(211-275 \mathrm{GHz}), 3584$ in band $7(275-373 \mathrm{GHz})$, 393 in band $8(385-500 \mathrm{GHz}), 220$ in band $9(602-720 \mathrm{GHz})$, and 8 in band $10(787-950 \mathrm{GHz})$.

The ALMA measurements of the 754 calibrators are included as supplementary material in the electronic version of the paper and on the website of the Italian ARC (http://arc.ia2.inaf.it). The catalogue gives the ALMA name, the source classification, its redshift (if available), the equatorial coordinates (J2000), the flux density measured in each observation with its error, the effective observing frequency, and the date and UTC time of the observation. The error is essentially given by the uncertainty in the flux density calibration (errors due to instrumental noise are typically smaller by more than two orders of magnitudes); we adopt a calibration uncertainty of 5 per cent (Fomalont private communication). ${ }^{3}$ An example of the content of the catalogue is given in Table 1. The coordinates are the average between the positions measured in the different ALMA observations.

We have recovered the redshifts of 589 sources $(\sim 78$ per cent of the total), using the Astroquery ${ }^{4}$ affiliated package of ASTROPY ${ }^{5}$ on the NASA/IPAC Extragalactic data base ${ }^{6}$ (NED), VizieR ${ }^{7}$, and $\mathrm{SIMBAD}^{8}$ data bases. Redshifts for 256 calibrators were provided by Mahony et al. (2011). Whenever multiple redshifts of the same source were found, we give the median value.

In Fig. 1, we show the Mollweide projection of the positions of

\footnotetext{
${ }^{2}$ We derived the flux densities through an image analysis instead of simply using model-fit values, because the former approach provides robust measurements for both resolved and unresolved sources, while model-fit flux densities are reliable for unresolved observations only.

${ }^{3}$ The debate about the precise value of the calibration uncertainty is still open in the ALMA community. Our results about the differences in flux density of the different calibrators for short timespans (see Section 4) support the adopted 5 per cent level.

${ }^{4}$ https://astroquery.readthedocs.io/en/latest/

${ }^{5} \mathrm{http} / / /$ www.astropy.org/

${ }^{6} \mathrm{https}: / /$ ned.ipac.caltech.edu/

${ }^{7}$ http://vizier.u-strasbg.fr/viz-bin/VizieR

${ }^{8} \mathrm{http} / / / \mathrm{simbad} . \mathrm{u}-\mathrm{strasbg.fr} / \mathrm{simbad} /$
} 
Table 1. Example of the catalogue content. The complete catalogue is available as supplementary material in the electronic version of the paper and on the website of the Italian ARC (http://arc.ia2.inaf.it)

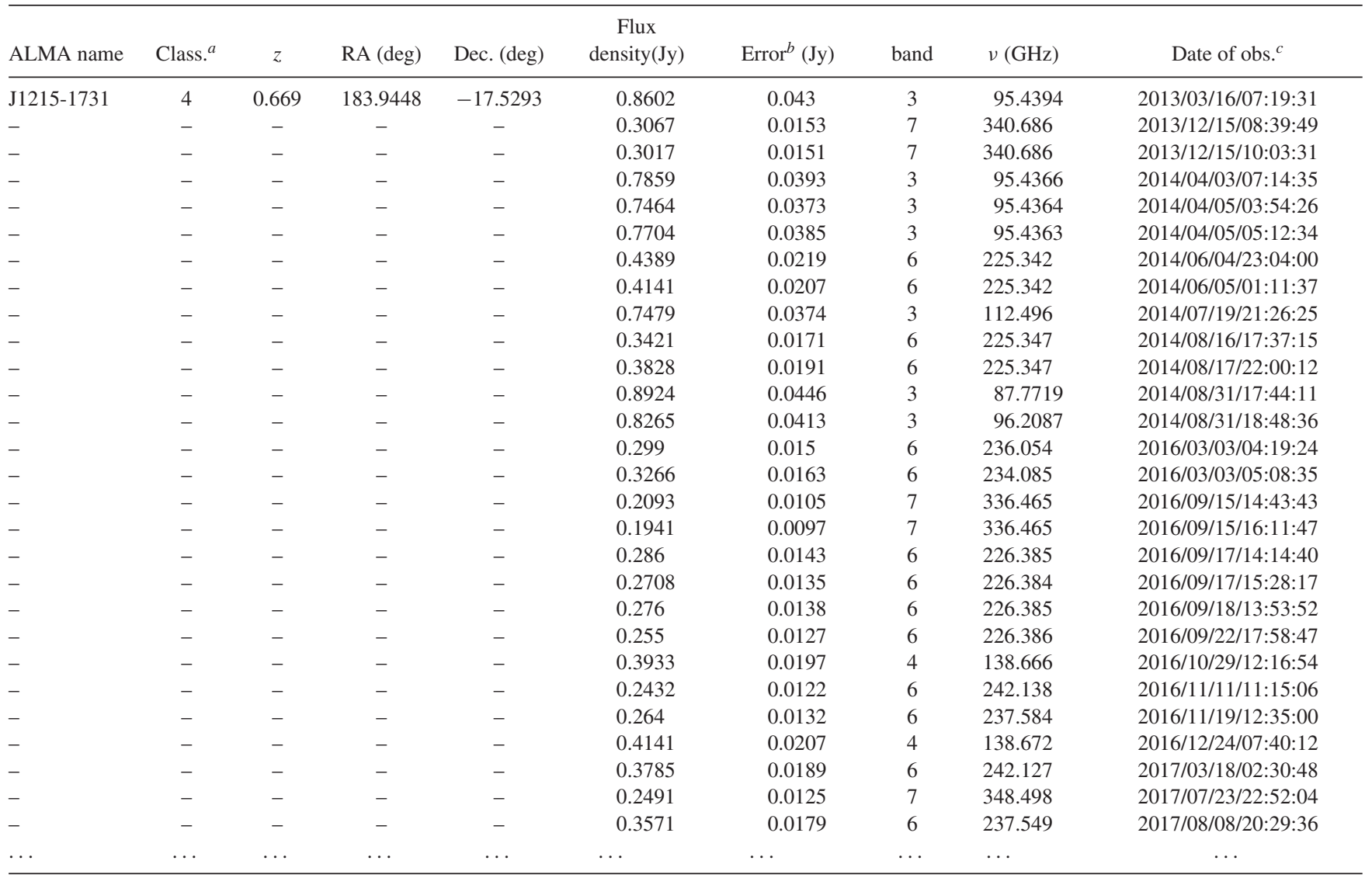

${ }^{a}$ Classification: 1 = FSRQ; $2=$ BLLac; $3=$ BLLac-galaxy dominated; $4=$ Blazar uncertain type; $5=$ BLLac candidate; $6=$ steep-spectrum; $7=$ uncertain.

${ }^{b}$ The uncertainty is given by summing in quadrature the rms and a typical ALMA calibration error equal to 5 per cent of the flux (see the text).

${ }^{c}$ Observing time in the format [YYYY/MM/DD/hh:mm:ss], UTC time.

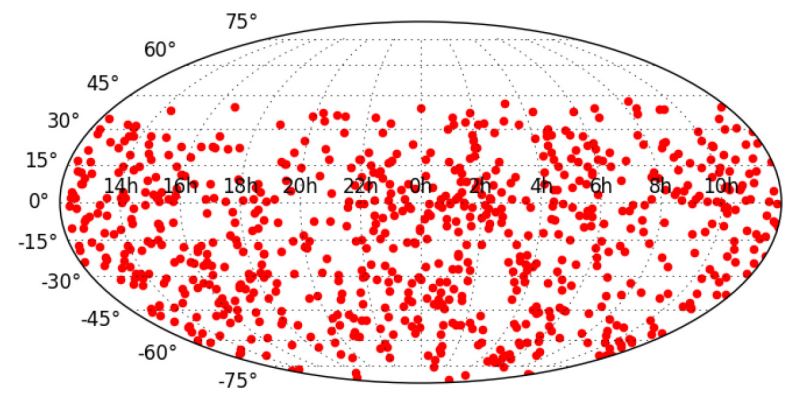

Figure 1. Mollweide plot showing the spatial distribution of the ALMACAL calibrators considered in this paper.

the ALMA calibrators, obtained through KAFE.

\section{SOURCE CLASSIFICATION}

Most sources of our catalogue (489, i.e. $\simeq 67$ per cent) are included in the 5th edition of the Roma Multi frequency Catalogue of Blazars ${ }^{9}$ (BZCAT; Massaro et al. 2009). BZCAT sources are divided into five

\footnotetext{
${ }^{9} \mathrm{http}: / /$ www.ssdc.asi.it/bzcat/
}

subclasses: FSRQs, BL Lacs, BL Lacs-galaxy dominated, Blazars of uncertain type, and BL Lac candidates.

We split the remaining 265 sources into the two classical subpopulations of steep-spectrum and flat-spectrum sources. As usual, such classification is based on the low-frequency (between $\simeq 1$ and $\simeq 5 \mathrm{GHz})$ spectral index, $\alpha_{\text {low }}$, adopting $\alpha_{\text {low }}=-0.5\left(S_{v} \propto v^{\alpha}\right)$ as the boundary value. The flat-spectrum population is essentially made by blazars.

We computed $\alpha_{\text {low }}$ using the $1.4 \mathrm{GHz}$ flux densities from the NRAO VLA Sky Survey (NVSS; Condon et al. 1998) complemented with those at $843 \mathrm{MHz}$ from the Sydney University Molonglo Sky Survey (Mauch et al. 2003), combined with those at $4.85 \mathrm{GHz}$ from the Green Bank $6 \mathrm{~cm}$ (Gregory et al. 1996) or from the Parkes-MIT-NRAO (Griffith \& Wright 1993) survey catalogues.

The low-frequency spectral index could be computed for all but 13 sources (out of 265) that were classified as 'uncertain'. Sources with $\alpha_{\text {low }}<-0.5$ were classified as steep-spectrum, provided they did not show clear variability or $\gamma$-ray emission. Only 10 sources satisfy the criteria for a steep-spectrum classification.

The overwhelming majority, 731 sources, i.e. $\sim 97$ per cent of the sample, are classified as blazars (since they belong to the BZCAT catalogue or they fulfill the criteria presented above). This includes also those with $\alpha_{\text {low }}<-0.5$ but with statistically significant variability and/or $\gamma$-ray emission (31 sources). We classify as 'Blazar uncertain type' our blazars without a BZCAT classification. 


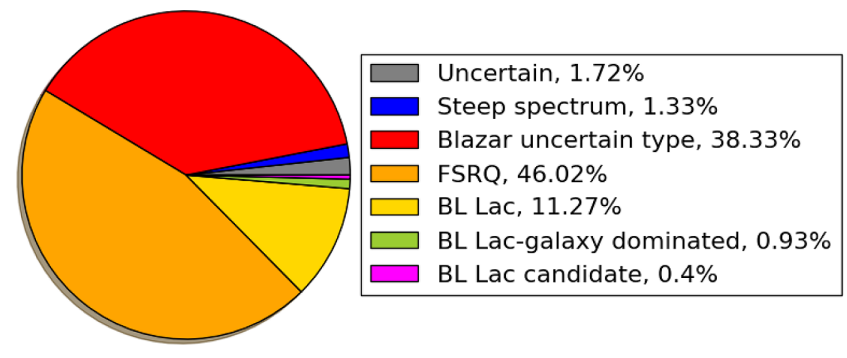

Figure 2. Pie chart showing the fractions of our ALMACAL calibrators in the different classes of classification (see Section 3).

The classification assigned to each source is given in the second column of Table 1. The pie chart (Fig. 2) illustrates the numerical proportions of sources in the different classes.

The classical physical models of blazars predict a steepening of their radio spectra at millimetre wavelengths (Kellermann 1966; Blandford \& Königl 1979). Statistical evidence of such steepening has been reported by several authors (González-Nuevo et al. 2008; Planck Collaboration XIII 2011; Planck Collaboration XV 2011; Planck Collaboration XLV 2016). The ALMA data allow us to check this prediction on a much larger sample than was possible before.

Tucci et al. (2011) went one step further. Their most successful physical evolutionary models of radio sources entail different distributions of break frequencies (the frequencies where the spectra steepen), for BL Lacs and FSRQs. They argue that BL Lacs have substantially higher break frequencies, implying that their synchrotron emission comes from more compact regions. Their best model, C2Ex, that successfully fits number counts and spectral index distributions of extragalactic radio sources over the $5-220 \mathrm{GHz}$ frequency range, predicts, for bright blazars $\left(S_{5 \mathrm{GHz}}>0.1 \mathrm{Jy}\right.$, like sources in our sample), that the break frequencies of most FSRQs are well below $100 \mathrm{GHz}$ while those of most BL Lacs are well above this frequency (cf. their Fig. 7). The ALMA data are well suited to test this prediction.

The left-hand panel of Fig. 3 shows the distribution of highfrequency spectral indices $\left(\alpha_{\text {high }}\right.$, from the effective frequencies of ALMA band 3 [84-116 GHz] to those of band 6 [211-275 GHz]) versus $\alpha_{\text {low }}$ for FSRQs and BL Lacs. The right-hand panel shows the distributions of such $\alpha_{\text {high }}$ indices.

Most low-frequency spectral indices are in the range from -0.5 to 0.8 , while most of the high-frequency ones range from -1.3 to 0 . Within these ranges, there is no correlation between the highand low-frequency spectral indices. The median spectral indices substantially steepen from low- to high-frequencies. For FSRQs, we have $\alpha_{\text {low, median }} \simeq 0.11$ (with first and third quartile values of about -0.10 and 0.29 , respectively) and $\alpha_{\text {high, median }} \simeq-0.65$ (first and third quartile values of about -0.85 and -0.51 , respectively). For BL Lacs $\alpha_{\text {low, median }} \simeq 0.05$ (first and third quartile values of about -0.18 and 0.22 , respectively) and $\alpha_{\text {high, median }} \simeq-0.48$ (first and third quartile values of about -0.61 and -0.33 , respectively). The global (FSRQ + BL Lac) median high-frequency spectral index $\alpha_{\text {high, median }} \simeq-0.63$ (with first and third quartile values of about -0.80 and -0.45 , respectively) is in good agreement with those found by Massardi et al. (2016) for the Planck-ATCA Co-eval Observations (PACO) bright sample: $\alpha_{\text {median, } 100-143 \mathrm{GHz}}=-0.67$ (with first and third quartile values of -0.94 and -0.45 , respectively); $\alpha_{\text {median, } 143-217 \mathrm{GHz}}=-0.57$ (with first and third quartile values of -0.83 and -0.45 , respectively).
There is thus evidence of a flatter median $\alpha_{\text {high }}$ of BL Lacs compared to FSRQs. The statistical significance of the difference was estimated using the two-sample Kolmogorov-Smirnov (KS) test, i.e. computing

$X^{2}=4 D^{2} \frac{m n}{m+n}$,

where $D$ is the KS statistics, which is the largest discrepancy between the cumulative distributions of high-frequency spectral indices of the two source populations, FSRQs and BL Lacs, comprising $m=117$ and $n=36$ sources, respectively.

We find $D=0.346$ corresponding to a 0.2 per cent probability that the two populations are drawn from the same parent distribution. A simpler, although less rigorous, illustration of the significance of the difference can be obtained considering that the ratio of the numbers of FSRQs in the bins $-1.1 \lesssim \alpha_{\text {high }} \lesssim-0.5$ and $-0.5 \lesssim$ $\alpha_{\text {high }} \lesssim 0$ is $\sim 2.4$ (the FSRQs in the two bins are $71+30=101$; see the right-hand panel of Fig. 3). If the $15+20=35$ BL Lacs were extracted from the same parent population, we would expect a similar ratio between the two bins, i.e. the expected number of BL Lacs in the first bin would be $71 \times(35 / 101) \sim 24.6$ and in the second bin would be $30 \times(35 / 101) \sim 10.4$. Based on the Poisson statistics, the probability of getting in the second bin 20 objects when 10.4 are expected is $\simeq 0.3$ per cent, close to the result of the KS test.

The statistically significant difference between the distributions of $\alpha_{\text {high }}$ for the two populations might be consistent with higher break frequencies for BL Lacs compared to FSRQs, as suggested by the Tucci et al. (2011) model. However, Planck Collaboration XLV (2016) did not find significant differences in the break frequencies of the two populations for their complete flux-density-limited sample of 104 extragalactic radio sources detected by the Planck satellite, but reported average spectral indices above the break frequency significantly steeper for FSRQs than for BL Lacs.

\section{PROPERTIES OF THE SAMPLE}

The flux density distributions of sources in the two most frequented ALMA bands (bands 3 and 6) are shown in Fig. 4. They extend from $\sim 1 \mathrm{mJy}$ to $\sim 15 \mathrm{Jy}$, with a peak at $\sim 0.2 \mathrm{Jy}$. For comparison, the minimum flux densities of sources in the 'extragalactic zone' $\left(|b|>30^{\circ}\right)$ listed in the Second Planck Catalogue of Compact Sources (PCCS2; Planck Collaboration XXVI 2016) are $232 \mathrm{mJy}$ at $100 \mathrm{GHz}$ and $127 \mathrm{mJy}$ at $217 \mathrm{GHz}$. Therefore, the ALMA observations reach much fainter flux densities than the Planck ones, but there is a large overlap between the two sets of observations.

As mentioned in Section 2, we have recovered redshift measurements for $\simeq 78$ per cent of our sample ( 589 sources). The redshift distributions of sources detected in bands 3 and 6 are shown in Fig. 5 . Both distributions peak at $0.5<z<1$ and have tails extending up to $z \sim 3.5$.

Fig. 6 shows the distribution of the number of observations of a given source, again in bands 3 and 6 , for sources with $\geq 2$ observations. The distributions peak at the lowest bin (2-8 measurements) but there is a significant number of sources with tens of measurements, up to $\sim 250$; sources with $\geq 20$ observations are 46 in band 3 and 52 in band 6 .

The timespan distribution of measurements in the source frame $\left(\Delta t_{\text {source }}=\Delta t_{\text {observer }} /(1+z)\right.$, where $\Delta t_{\text {observer }}$ is the time between the first and the last observation) is shown in Fig. 7. We excluded all the sources lacking redshift measurements. Although for most sources the timespan is relatively short (less than a few hundreds 

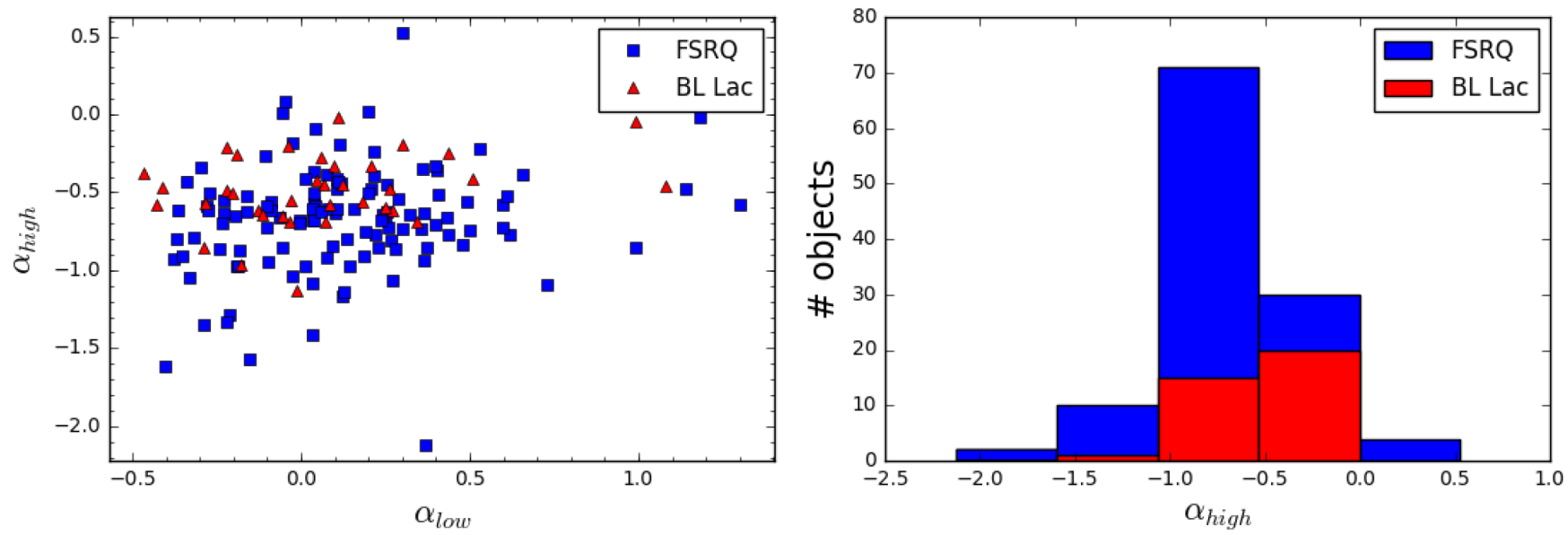

Figure 3. On the left, high- versus low-frequency spectral indices of FSRQs and BL Lacs of our sample. $\alpha_{\text {low }}$ is calculated between $1.4 \mathrm{GHz}$ (or $0.84 \mathrm{GHz}$ for sources outside the NVSS area) and $4.8 \mathrm{GHz} ; \alpha_{\text {high }}$ is between the effective frequencies (listed in Table 1) in ALMA 3 and in ALMA 6 bands. The right-hand panel shows the distribution of $\alpha_{\text {high }}$ for the two populations.

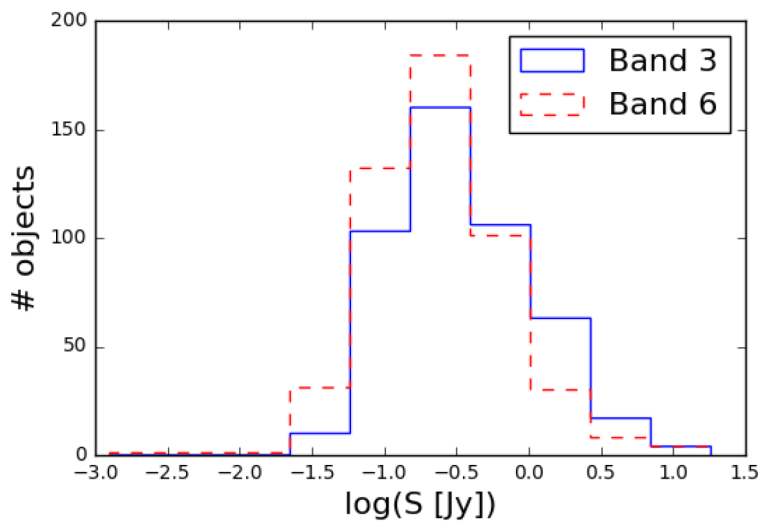

Figure 4. Flux density distributions of sources detected in ALMA band 3 (solid blue line) and band 6 (dashed red line).

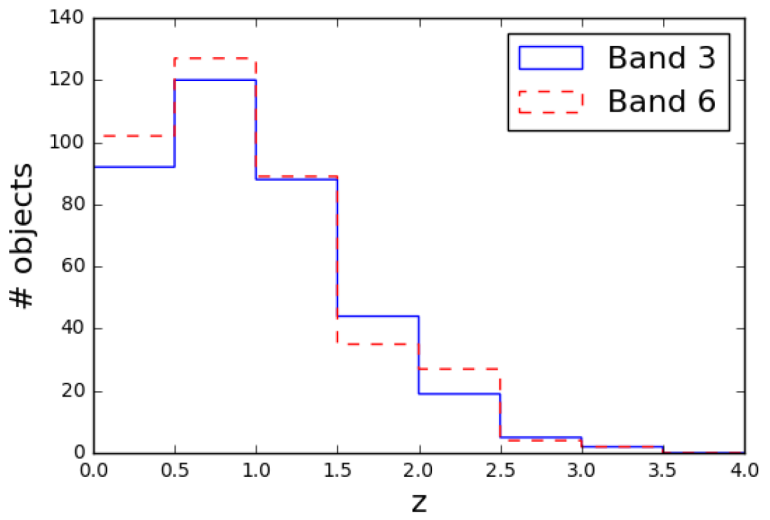

Figure 5. Redshift distributions of the sources detected in ALMA band 3 (solid blue line) and band 6 (dashed red line).

of days), for some sources observations cover a few years (in the source frame); sources with $\Delta t_{\text {source }} \geq 2 \mathrm{yr}$ are 20 in band 3 and 28 in band 6 .

The light curves in bands 3 and 6 of three of the most frequently observed sources are shown in Fig. 8. Monitoring of blazars is important to understand which mechanisms drive their violent

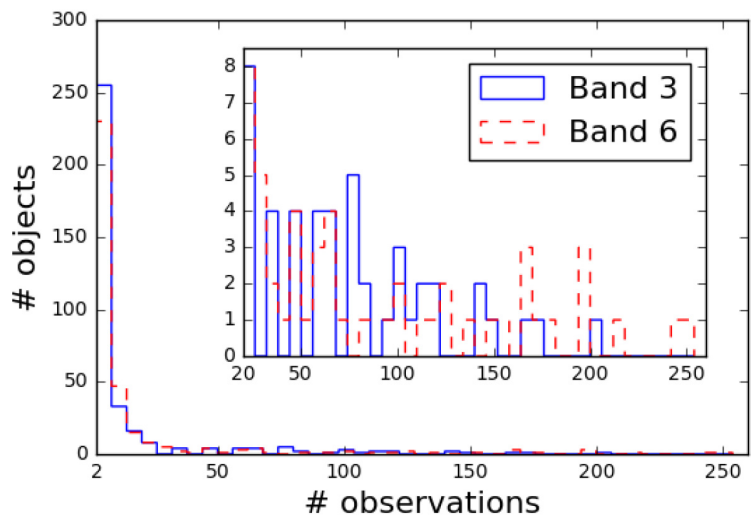

Figure 6. Distributions of the number of observations per source in ALMA band 3 (solid blue line) and in band 6 (dashed red line); bin width $=6$. We only considered sources with $\geq 2$ observations. In the zoomed-up inset plot, we show the portion of $\geq 20$ observations only.

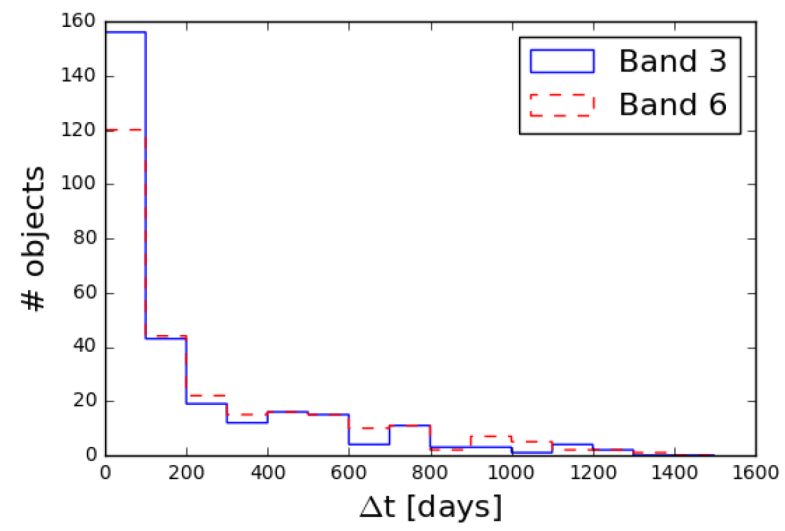

Figure 7. Distributions of the rest-frame timespans of source observations (i.e. $\left.\Delta t=\left[t_{\text {last observation }}-t_{\text {first observation }}\right] /[1+z]\right)$ in ALMA band 3 (solid blue line) and in band 6 (dashed red line). We only considered sources having redshift measurements and $\geq 2$ observations. 

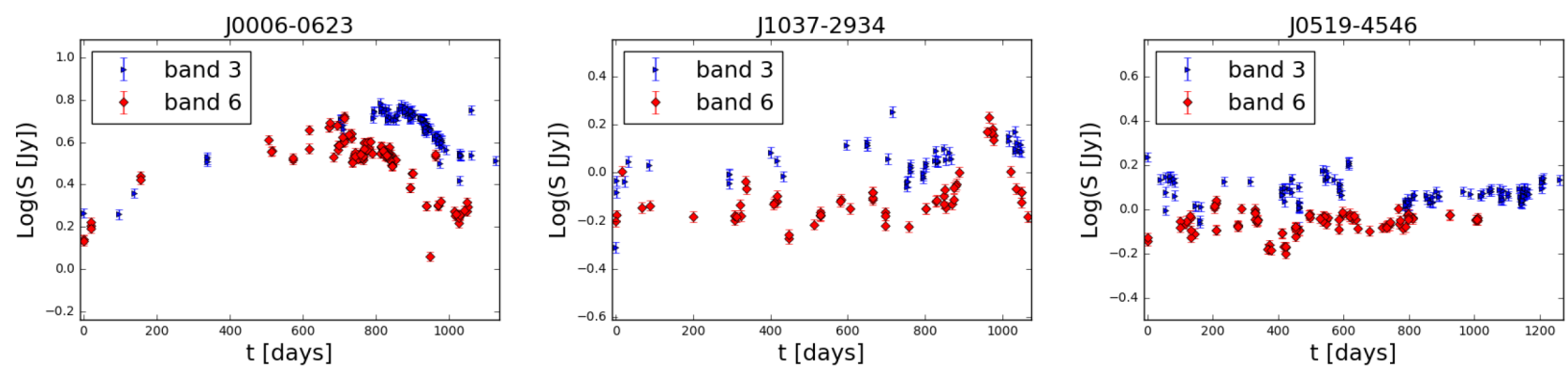

Figure 8. Light curves of three of the most frequently observed sources in our sample. The time is in the source frame. All three sources show significant variability but with remarkably different flare morphology. In the case of J0006-0623, ALMA observations have monitored the entire duration of a big flare, lasting for years, showing that it had a similar amplitude in band 6 and in band 3 but a shorter duration in band 6. J1037-2934 shows, in both bands, a sequence of relatively short duration, moderate amplitude flares, the last of which, best monitored in band 6 , is the most prominent one. The rise times of the flares are generally shorter than the decay times, consistent with the results by Nieppola et al. (2009). Also J0519-4546 shows a sequence of moderate amplitude, short flares, but they are followed by a relatively quiescent period.

variability and what is the duty cycle of their activity. The interest on multi frequency blazar monitoring has gained momentum since the launch of the Fermi Gamma-ray Space Telescope. In fact, the overwhelming majority of detected extragalactic $\gamma$-ray sources are blazars, and Fermi has gathered spectacular $\gamma$-ray light curves of hundreds of them (Abdo et al. 2010; Abdollahi et al. 2017). The poorly explored few-mm to sub-mm spectral region covered by ALMA observations is important in this context since flux densities in this region appear to be particularly well correlated with those at $1 \mathrm{GeV}$ (Fuhrmann et al. 2016).

An obvious use of multiple observations is the calculation of the variability index (VI), defined as (Sadler et al. 2006)

$V I=\frac{100}{\langle S\rangle} \times \sqrt{\frac{\sum\left[S_{i}-\langle S\rangle\right]^{2}-\sum\left(\sigma_{i}\right)^{2}}{N}}$,

where $S_{i}$ and $\sigma_{i}$ are the flux density measurements of a source measured in a given band and the associated uncertainties, $N$ is the number of measurements, and $\langle S\rangle$ is the mean flux density.

Obviously, the variability index can be reliably measured only if the amplitude of flux density variations is substantially larger than the 5 per cent calibration uncertainty, although equation (2) gives values of the variability index $<5$ per cent. Reliable variability indices are measured for 31/41,30/33, 29/33, and 26/26 (band 3) and 25/30,36/39,35/39, and 37/37 (band 6) sources for timespans of $100,200,400$, and $800 \mathrm{~d}$ (within \pm 30 per cent, in the source frame), respectively. All sources with $\geq 2$ measurements on these time-scales are included.

In Table 2 the median, first quartile, and third quartile values of the VI for the different source frame time-scales and in the two different bands are listed.

Applying the two-sample KS test to the VI distributions of the 100 and $800 \mathrm{~d}$ of time-scales, we find that the probability that the two subsamples are drawn from the same parent distribution is extremely low ( $<0.1$ per cent in both bands, with $D=0.588$ in band 3 and $D=0.732$ in band 6). This is a direct consequence of the fact that the characteristic time-scale of blazar variability in blazar sources is $\sim 3$ yr (see e.g. Nieppola et al. 2009).

Measured flux densities of the same source for short timespans are expected to be only weakly affected by variability. Differences among such measurements are therefore an estimator of systematic errors that afflict our observations and primarily of the calibration error. The median absolute values of differences among measurements in bands 3 and 6 done within $30 \mathrm{~d}$ in the source frame are $\simeq 4$ per cent for band 3 and $\simeq 5$ per cent for band 6 , consistent with the adopted calibration error of 5 per cent.

\section{GLOBAL SPECTRAL ENERGY DISTRIBUTIONS OF SOURCES}

As mentioned in Section 3, our source classification is based on external data. For each source in our sample, we have collected the photometric data available on the NED using Astroquery with a search radius of $10 \mathrm{arcsec}$, excluding Galactic sources.

The NED data were complemented by cross matching our catalogue with the Australia Telescope 20 GHzSurvey Catalogue (AT20G; Murphy et al. 2010), the PCCS2 (Planck Collaboration XXVI 2016), the PACO catalogue (Massardi et al. 2016), the Galactic and Extragalactic All-sky MWA survey (GLEAM; HurleyWalker et al. 2017), and the CRATES survey (Healey et al. 2007).

The cross matching was done using the following search radii: 5 arcsec around the ALMA positions for the AT20G catalogue; $16,13.5,6.5,4.85,3.6,2.45,2.45,2.35$, and 2.1 arcmin (i.e. half FWHM) for the 30, 44, 70, 100, 143, 217, 353, 545, and $857 \mathrm{GHz}$ PCCS2 catalogues, respectively; 20 arcsec for the GLEAM catalogue; 70 arcsec for the CRATES one. For the PACO catalogue, we exploited the AT20G identifications by Massardi et al. (2016). We considered PCCS2 data only for sources with $|b|>10^{\circ}$ to avoid wrong identifications (with Galactic sources). In all the cases, the search yielded a unique identification.

In this way, we obtained SEDs extending over 17 orders of magnitude, from radio to $\gamma$-rays. The complete SED collection is available on the website of the Italian ARC (http://arc.ia2.inaf.it). Some examples are shown in Fig. 9. We found $\gamma$-rays measurements for 248 sources ( $\sim 33$ per cent of the sample).

\section{SUMMARY}

We have presented a catalogue of ALMA flux density measurements of calibrators, observed between 2012 August and 2017 September, in the framework of the ALMACAL project. The ALMACAL images were reprocessed using a new code developed by the Italian node of the European ARC. This has yielded 16263 flux density measurements in different ALMA bands and at different epochs of 754 calibrators. A search in online data bases has yielded redshifts for 589 sources ( $\sim 78$ per cent of the total).

Most (489, i.e. $\simeq 67$ per cent) of our sources are classified as blazars of various types in the BZCAT catalogue. Almost all of 

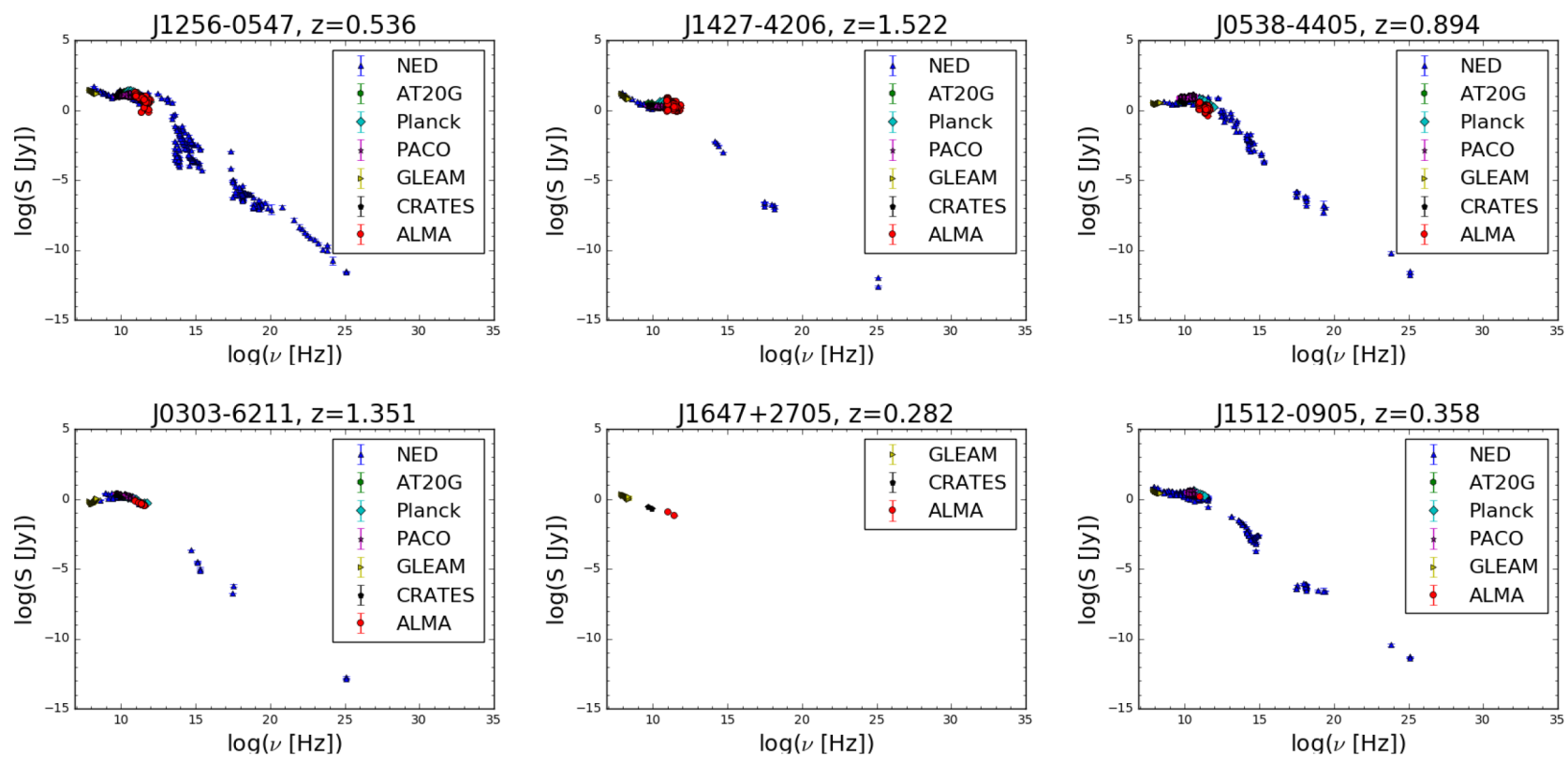

Figure 9. Examples of SEDs of our sample reconstructed using the collection of photometric data described in Section 5: 'NED' from https://ned.ipac.c altech.edu/; 'AT20G' from Murphy et al. (2010); 'PCCS2' from Planck Collaboration XXVI (2016); 'PACO' from Massardi et al. (2016); 'GLEAM' from Hurley-Walker et al. (2017); 'CRATES' from Healey et al. (2007); 'ALMA' are the new observations presented in this paper. The complete SED collection of our sample is available on the website of the Italian ARC (http://arc.ia2.inaf.it).

Table 2. Median, first quartile, and third quartile values of the variability indices for four different source frame timespans (100, 200, 400, and $800 \mathrm{~d}$ ) in ALMA band 3 and 6.

\begin{tabular}{lcccc}
\hline Band & $\begin{array}{c}\text { Timespan } \\
(\mathrm{d})\end{array}$ & $\begin{array}{c}\mathrm{VI}_{\text {median }} \\
\text { (per cent) }\end{array}$ & $\begin{array}{c}\mathrm{VI}_{1^{s t}} \text { quartile } \\
\text { (per cent) }\end{array}$ & $\begin{array}{c}\mathrm{VI}_{3^{r d}} \text { quartile } \\
\text { (per cent) }\end{array}$ \\
\hline 3 & 100 & 5.9 & 1.7 & 11.8 \\
& 200 & 12.8 & 5.7 & 24.3 \\
& 400 & 14.2 & 4.5 & 24.8 \\
6 & 800 & 23.9 & 13.9 & 32.8 \\
& 100 & 8.1 & 4.4 & 12.2 \\
& 200 & 11.9 & 7.0 & 20.2 \\
& 400 & 14.3 & 8.1 & 25.6 \\
& 800 & 21.3 & 18.6 & 33.4 \\
\hline
\end{tabular}

the remaining sources have properties (flat low-frequency radio spectrum, clear variability in different bands, and $\gamma$-ray emission) consistent with a blazar classification. In total, $\sim 97$ per cent of the sources are classified as blazars.

To illustrate the properties of the sample, in view of its exploitation for scientific investigations, we have focused on the most frequented ALMA bands, i.e. bands 3 and 6. For these bands, we have shown the redshift and flux density distributions of catalogued sources, the distribution of the number of observations of individual sources, and of timespans in the source frame.

Several sources have tens of measurements in a band, covering several years. As an example of the variety of scientific investigations allowed by the catalogue, we have presented unprecedented band 3 and 6 light curves of three sources and estimates of the variability indices on time-scales of 100, 200, 400, and $800 \mathrm{~d}$ in the same bands.

Through an analysis of flux density differences for short timespans, in bands 3 and 6, we have found that the systematic errors are consistent with the adopted calibration error of 5 per cent.
We have also found that the ALMA data show highly significant evidence of a difference between the high-frequency $(v \gtrsim 100 \mathrm{GHz})$ spectra of FSRQs and BL Lacs: at wavelengths a few $\mathrm{mm}$, the average spectra of BL Lacs are flatter than those of FSRQs. This is expected if the synchrotron emission of BL Lacs comes from more compact regions than the emission of FSRQs, as argued, e.g. by Tucci et al. (2011).

Finally, by collecting data from online data bases, we have reconstructed the SEDs of our sources over 17 orders of magnitude in frequency. Both the catalogue and the SEDs are available to the community.

\section{ACKNOWLEDGEMENTS}

We thank Ian Smail for his useful comments. We are indebted to Edward Fomalont for enlightening discussions, highly valuable suggestions, and for his extensive work, together with Ruediger Kneissl and Antonio Hales, on ALMA calibrator catalogues and calibration error estimation. This paper and the AKF and KAFE development are part of the activities for the ALMA re-imaging Study approved in the framework of the 2016 ESO Call for Development Studies for ALMA Upgrade (PI: Massardi). The study acknowledges partial financial support by the Italian Ministero dell'Istruzione, Università e Ricerca through the grant 'Progetti Premiali 2012 iALMA' (CUP C52I13000140001). GDZ acknowledges support from ASI/INAF agreement no. 2014-024-R. 1 for the Planck LFI Activity of Phase E2 and from the ASI/Physics Department of the university of Roma-Tor Vergata agreement no. 2016-24-H.0 for study activities of the Italian cosmology community. MN acknowledges support from the European Union's Horizon 2020 research and innovation programme under the Marie Skłodowska-Curie grant agreement no. 707601. This research has made use of the NED, which is operated by the Jet Propulsion Laboratory, California Institute of Technology, under contract with the National Aeronautics and Space Administration. 


\section{REFERENCES}

Abdo A. A. et al., 2010, ApJ, 722, 520

Abdollahi S. et al., 2017, ApJ, 846, 34

Blandford R. D., Königl A., 1979, ApJ, 232, 34

Burkutean S. et al., 2018, J. Astron. Telesc. Instrum. Syst., 4, 028001

Condon J. J., Cotton W. D., Greisen E. W., Yin Q. F., Perley R. A., Taylor G. B., Broderick J. J., 1998, AJ, 115, 1693

Fomalont E. et al., 2014, The Messenger, 155, 19

Fuhrmann L. et al., 2016, A\&A, 596, A45

González-Nuevo J., Massardi M., Argüeso F., Herranz D., Toffolatti L., Sanz J. L., López-Caniego M., de Zotti G., 2008, MNRAS, 384, 711

Gregory P. C., Scott W. K., Douglas K., Condon J. J., 1996, ApJS, 103, 427 Griffith M. R., Wright A. E., 1993, AJ, 105, 1666

Healey S. E., Romani R. W., Taylor G. B., Sadler E. M., Ricci R., Murphy T., Ulvestad J. S., Winn J. N., 2007, ApJS, 171, 61

Hurley-Walker N. et al., 2017, MNRAS, 464, 1146

Kellermann K. I., 1966, ApJ, 146, 621

Klitsch A., Peroux C., Zwaan M. A., Smail I., Oteo I., Biggs A. D., Popping G., Swinbank A. M., 2017, MNRAS, 475, 492,

Liuzzo E. et al., 2018, ALMA memo, submitted

Mahony E. K. et al., 2011, MNRAS, 417, 2651

Massardi M., Bonaldi A., Bonavera L., De Zotti G., Lopez-Caniego M., Galluzzi V., 2016, MNRAS, 455, 3249

Massaro E., Giommi P., Leto C., Marchegiani P., Maselli A., Perri M., Piranomonte S., Sclavi S., 2009, A\&A, 495, 691

Mauch T., Murphy T., Buttery H. J., Curran J., Hunstead R. W., Piestrzynski B., Robertson J. G., Sadler E. M., 2003, MNRAS, 342, 1117
Murphy T. et al., 2010, MNRAS, 402, 2403

Nieppola E., Hovatta T., Tornikoski M., Valtaoja E., Aller M. F., Aller H. D., 2009, AJ, 137, 5022

Oteo I., Zwaan M. A., Ivison R. J., Smail I., Biggs A. D., 2016, ApJ, 822, 36

Oteo I., Zwaan M. A., Ivison R. J., Smail I., Biggs A. D., 2017, ApJ, 837, 182

Planck Collaboration XIII, 2011, A\&A, 536, A13

Planck Collaboration XLV, 2016, A\&A, 596, A106

Planck Collaboration XV, 2011, A\&A, 536, A15

Planck Collaboration XXVI, 2016, A\&A, 594, A26

Sadler E. M. et al., 2006, MNRAS, 371, 898

Tucci M., Toffolatti L., de Zotti G., Martínez-González E., 2011, A\&A, 533, A57

\section{SUPPORTING INFORMATION}

Supplementary data are available at MNRAS online.

Please note: Oxford University Press is not responsible for the content or functionality of any supporting materials supplied by the authors. Any queries (other than missing material) should be directed to the corresponding author for the article.

This paper has been typeset from a $\mathrm{T}_{\mathrm{E}} \mathrm{X} / \mathrm{L} \mathrm{T} \mathrm{E} \mathrm{X}$ file prepared by the author. 